\title{
Implementation of a neonatal pain management module in the computerized physician order entry system
}

Nathalie Mazars ${ }^{1 \dagger}$, Christophe Milési $^{1}$, Ricardo Carbajal ${ }^{2,3}$, Renault Mesnage ${ }^{1}$, Clémentine Combes ${ }^{1}$, Aline Rideau Batista Novais ${ }^{1}$ and Gilles Cambonie ${ }^{1,4^{*}}$

\begin{abstract}
Background: Despite the recommended guidelines, the neonatal management of pain and discomfort often remains inadequate. The purpose of the present study was to determine whether adding a pain and discomfort module to a computerized physician order entry (CPOE) system would improve pain and discomfort evaluation in premature newborns under invasive ventilation.

Methods: All newborns <37 weeks gestational age (GA) and requiring invasive ventilation were included in a prospective study during two 6-month periods: before and after the inclusion of the pain and discomfort evaluation module. The main outcome measure was the percentage of patients having at least one assessment of pain and discomfort per day of invasive ventilation using the COMFORT scale.

Results: A total of 122 patients were included: 53 before and 69 after the incorporation of the module. The mean age was 30 (3) weeks GA. After the module was included, the percentage of patients who benefited from at least one pain and discomfort assessment per day increased from $64 \%$ to $88 \%(p<0.01)$, and the mean number (SD) of scores recorded per day increased from 1 (1) to $3(1)(p<0.01)$. When the score was not within the established range, the nursing staff adapted analgesia/sedation doses more frequently after module inclusion (53\% vs. 34\%, $p<0.001)$. Despite higher mean doses of midazolam after module introduction [47 (45) vs. 31 (18) $\mu \mathrm{g} / \mathrm{kg} / \mathrm{hr}$, $p<0.05]$, the durations of invasive ventilation and hospital stay, and the number of nosocomial infections, were not significantly modified.
\end{abstract}

Conclusions: Adding a pain and discomfort tool to the CPOE system was a simple and effective way to improve the systematic evaluation of premature newborns who required ventilatory assistance.

Keywords: Analgesia, Computer-assisted instruction, Newborn, Pain management, Sedation

\section{Background}

Premature newborns hospitalized in intensive care undergo many painful medical acts, with some studies signaling an average of 15 such acts per day [1,2]. Managing the pain and discomfort (PAD) of these infants is a therapeutic priority because of the immediate consequences to

\footnotetext{
*Correspondence: g-cambonie@chu-montpellier.fr

${ }^{\dagger}$ Equal contributors

${ }^{1}$ Neonatology Department, Arnaud de Villeneuve Hospital, CHU Montpellier, Montpellier F-34000, France

${ }^{4}$ Neonatology and Intensive Care Unit, Montpellier University Hospital Centre, Arnaud de Villeneuve Hospital, 371 Avenue du Doyen G Giraud, 34295,

Montpellier, Cedex 5, France

Full list of author information is available at the end of the article
}

the infants' stability [3] and the long-term repercussions on neuroendocrine development and the capacity to manage stress from nociceptive stimuli [4-6].

Although the message that relieving and preventing PAD in newborns has been widely disseminated to all concerned medical staff, PAD management remains inadequate $[7,8]$. In 2006, a rigorous analysis of the literature suggested that these practices could be improved by establishing precise objectives, including the systematic evaluation of PAD and the development and formalization of protocols for its management $[9,10]$.

On our unit, many actions have been initiated since 2002: the creation of a "pain group," biannual training 
courses about pain evaluation and treatment, placement of COMFORT scales in front of each incubator, and the construction of a pain management protocol available in paper and electronic form. However, during a recent audit on PAD management in our NICU, we found that the most important objective we needed to reach was the systematization of PAD evaluation. We observed that only two thirds of the neonates under invasive ventilatory assistance had benefited from at least one formalized evaluation per day; that is, with the use of a checklist. This situation prompted us to develop a software module to specifically manage PAD, which was included in our daily-use computerized physician order entry (CPOE) system. We chose this tool for two reasons. First, we assumed that the module would encourage physicians to become more directly involved in pain management, because they would have to validate an algorithm daily to evaluate PAD and adapt analgesic/ sedative treatment, based on the unit protocol. Second, we assumed that this tool would encourage the nurses to score the pain objectively, because by doing so they would be able to adjust the analgesic/sedative doses on the basis of their evaluation.

The purpose of this study was to evaluate whether the inclusion of a PAD evaluation module in the NICU CPOE system would be an effective strategy to improve PAD evaluation in premature newborns requiring invasive ventilation.

\section{Methods}

This prospective, before-after study was conducted in the 12-bed NICU of a tertiary care university hospital in Montpellier, France. The ratio of pediatric nurses to infants is 1 to 2 and the shift rotation is every 12 hours.

\section{Local protocol for managing pain and discomfort}

Since 2002, our NICU staff has followed the protocol that we collectively developed for managing pain and discomfort; this protocol can be consulted in paper form in the NICU protocol binder. A printed version of the COMFORT scale for evaluating pain and discomfort is included in all patient charts, and all medical and paramedical personnel receive training in pain and discomfort evaluation and management twice a year.

\section{COMFORT scale}

The COMFORT scale assesses several components of pain and discomfort and has been validated in premature newborns under ventilation [11,12]. The components are essentially observable behaviors and variations in physiological criteria. The sum of the different items ranges from 8 to 40. Different cutoff or range values for the COMFORT scale have been proposed or established to describe the infant's status in terms of pain and discomfort $[13,14]$. On our unit, we follow the recommendations of the Centre National de Ressources de lutte contre la douleur (CNED; National Center for Resources to Combat Pain; http://www.pediadol.org/IMG/pdf/ COMFORT.pdf). Newborns are assumed to be "painfree and comfortable" with scores between 18 and 23 . They are considered to be "in pain or uncomfortable" with scores between 24 and 40, and "excessively sedated" with scores between 8 and 17. The interrater reliability of the COMFORT scale was verified when the scale was introduced on the NICU in 2002, but it was not repeated before beginning the study.

\section{NICU protocol}

The protocol remained the same and was presented to all staff before each phase of the study. All newborns under invasive ventilation received an opioid (sufentanil, starting dose: $0.1 \mu \mathrm{g} / \mathrm{kg} / \mathrm{hr}$ ) in combination with a benzodiazepine (midazolam, starting dose: $30 \mu \mathrm{g} / \mathrm{kg} / \mathrm{hr}$ ). The efficacy was evaluated every 8 hours by a pediatric nurse using the COMFORT scale and every 4 hours if a painful procedure had been performed. The objective was a score between 18 and 24. For a score $>24$, the first step was to determine whether there was a specific cause, such as product perfusion or respiratory obstruction, and to improve the environmental conditions, notably by changing the baby's position and the respirator settings if necessary. Then, if the excessively high COMFORT score was judged to be directly painrelated-for example, following a painful medical act-a bolus corresponding to an hourly dose of sufentanil was administered over $10 \mathrm{~min}$ and the hourly dose was increased by $20 \%$ if the score remained elevated after two boluses. If the excessively high COMFORT score was judged to be the result of discomfort-for example, a hyperalert newborn without a recent medical act or stimulation who seemed to be struggling against the respirator-the hourly dose of midazolam was increased by $20 \%$. If the score was $<18$, we began reducing the hourly dose of analgesia/sedation by $20 \%$ over 8 hours, usually beginning with the midazolam. All the patients also were managed according to the Neonatal Individualized Development Care and Assessment Program used on our NICU since 2004 [15].

\section{Computerized pain evaluation module}

A CPOE system was introduced on our unit in 2001 to limit the risk of medical errors [16]. The module was added to the CPOE system in such a way that, each time the software was run, a specific window opened first. Every day, this window prompted the physician to order COMFORT scale assessment at a modifiable frequency and the adaptation of sufentanil and/or midazolam 
dosages in line with the protocol. The physician then discussed the situation with the nurse in charge of the infant and sometimes modified the orders and validated them. The orders were then printed out, with the individualized PAD management algorithm added to the other medication and surveillance orders for the day (Figure 1). All of the therapeutic adaptations were thus carried out by the nurses according to the algorithm. The physician was notified if the increase or decrease in dosages or bolus administration did not lead to normalization of the COMFORT score in the ensuing 30 minutes.

\section{Population}

All premature newborns with $\mathrm{GA}<37$ weeks and requiring invasive mechanical ventilation were eligible for study inclusion. The noninclusion criteria were the following: a decision to limit or stop life-sustaining therapy and the use of curare during invasive ventilation.

\section{Study design}

The patients were prospectively enrolled in the study during two 6-month periods separated by the introduction of the computerized module for PAD evaluation and the adaptation of analgesic/sedative dosages based on the score. Two groups of patients were compared.

\section{Control group}

The control group was recruited during the period before the introduction of the PAD module, between September 2008 and February 2009. The orders to evaluate PAD and then modify the medication dosages on the basis of the score followed the protocol established in our unit; this protocol was available to all staff but could be adapted by each prescribing physician.

\section{Intervention group}

This group of patients was recruited after the introduction of the PAD evaluation module on March 1, 2009. It

\section{Module filled in daily by the physician: $\quad$ Patient XX Weight: $1500 \mathrm{~g}$}

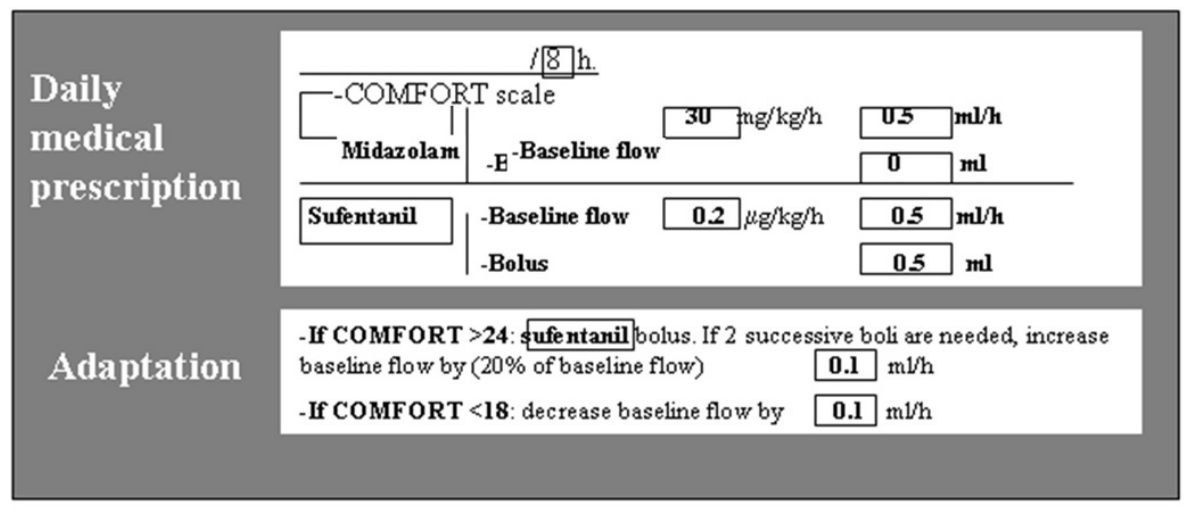

Part of the prescription for pain management printed and given to the nurse:

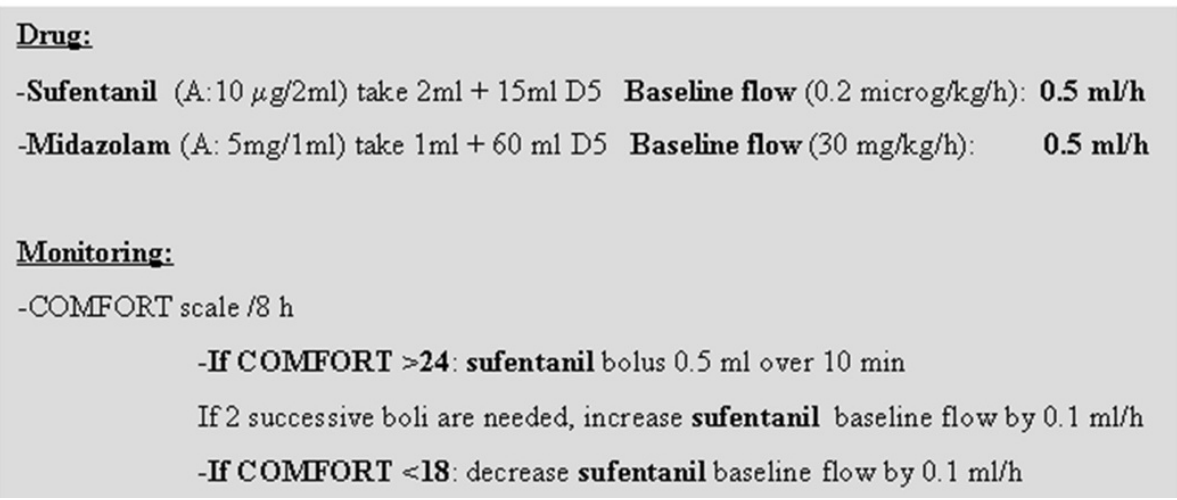

Figure 1 Example of the specific window for pain and discomfort evaluation and management. The window was activated during the entry of computerized physician orders and the retranscription printed out and given to the nurse. The physician could modify the frequency of evaluation with the COMFORT scale, and the baseline dosage and bolus dosages of analgesic and/or sedative. 
thus included the patients in the immediate postintervention period from March to August 2009. During this period, the protocol for PAD management remained the same on the NICU, notably regarding the nurses, who were able to adapt the analgesic/sedation dosages based on the COMFORT scores. The only modification in this period was the systematization of PAD assessment as part of the written orders given to them.

\section{Criteria for weaning from invasive ventilation}

Newborns were extubated when they presented all of the following criteria: 1) clinical stability and spontaneous autonomous ventilation on the endotracheal tube verified during routine care, such as changing diapers or modifying position; 2) positive expiratory pressure $<4 \mathrm{~cm} \mathrm{H}_{2} \mathrm{O}$ and maximal inspiratory pressure $<20 \mathrm{~cm} \mathrm{H} \mathrm{H}_{2} \mathrm{O}$ and $\mathrm{FiO}_{2}<0.4$; and 3) $\mathrm{pH}>7.25$ and $\mathrm{SpO}_{2}>85 \%$ [17]. Infants $<28$ weeks were systematically placed on nasal continuous airway pressure after the weaning from invasive ventilation.

\section{Prevention of withdrawal syndrome}

Once the decision to wean the infant from invasive ventilation was made, the NICU protocol called for a daily $20 \%$ reduction in the drug dosage when analgesic/sedatives had been given for more than 5 days and 10\% when the drugs had been given for more than 10 days. In addition, withdrawal syndrome was systematically evaluated by the neonatal abstinence score of Finnegan [18].

\section{Data collection}

The nurse in charge of the infant noted the exact time of PAD assessment and the therapeutic response (name of the drug, dose, and adaptation) in the patient chart. The main outcome measure was the percentage of patients having at least one evaluation per day of ventilation using the COMFORT scale, i.e., the ratio of the number of patients having at least one evaluation per day to the number of patients ventilated. During the ventilation period, if one of the daily nursing forms for hourly surveillance did not have a COMFORT score recorded, the infant was not included in the numerator but was included in the denominator. We arbitrarily chose the compliance rate of only one evaluation per day of ventilation in the absence of firm recommendations on the rate of evaluation in these patients.

The secondary outcome measures were the number of scores prescribed and assessed per day of ventilation, the mean COMFORT score, the mean dosages of analgesics and sedatives, the duration of ventilation, the duration of hospitalization, and the number of nosocomial infections.

\section{Statistical analysis}

To calculate the number of subjects needed, previous data collections suggested that the prevalence of at least a daily evaluation of the COMFORT score in the control group would be $65 \%$. Given an alpha risk of $5 \%$ and a power of $80 \%$, 50 patients per group were needed to show an increase in this prevalence, from $65 \%$ before module inclusion to $90 \%$ after, corresponding to a $38 \%$ improvement in scoring. Considering the mean number of premature infants admitted to our NICU for invasive ventilation, we chose a 6-month inclusion period for both the pre- and postintervention phases.

Summary statistics are given as means (SD) for continuous variables and as numbers (percentages) for categorical variables. Categorical variables were compared with the chi-squared test or Fisher's exact test when necessary. For continuous variables, group comparisons were made with Student's $t$ test or the Mann-Whitney when distributions were not normal or the variances were unequal. Statistical significance was set at 5\% for all tests. Statistical analysis was conducted with the SAS software package, version 9 (SAS Institute, Cary, NC).

\section{Ethical considerations}

The parents of all children gave written, informed consent to care. The procedures described herein are all part of routine care on our unit and did not require approval of the institutional review board, in line with French law.

\section{Results \\ Population}

From September 2008 to August 2009, 349 newborns $<37$ weeks GA were admitted to the NICU; 214 of them did not require invasive mechanical ventilation. Of the 135 who required invasive ventilatory assistance, 10 were excluded from analysis following the decision to limit or stop life-sustaining therapy and 3 because of missing data. No patient was treated with curare. Thus, 122 patients were included: 53 in the control group and 69 in the intervention group.

\section{Patient characteristics (Table 1)}

\section{Evaluation and management of PAD before and after the} computerized pain module inclusion (Tables 2 and 3)

The percentage of patients having at least one evaluation per day of ventilation using the COMFORT scale increased from $64 \%$ before module inclusion to $88 \%$ after inclusion $(p=0.002)$.

The mean number (SD) of COMFORT scores ordered per newborn and per day of ventilation also increased from 1 (2) before the intervention to 3 (2) after intervention $(p<0.01$, Figure 2).

The COMFORT scores fell outside the target range 182 times of the 813 scores collected before the 
Table 1 Clinical characteristics of patients, before (Control) and after (Intervention) inclusion of the module for evaluation and management of pain and discomfort

\begin{tabular}{lcccc}
\hline & Control & & Intervention & \\
\cline { 2 - 3 } & $\mathbf{n = 5 3}$ & & $\mathbf{n = 6 9}$ & $\boldsymbol{p}$ \\
\hline Gestational age, wk & $29(3)$ & & $30(3)$ & 0.07 \\
\hline Birth weight, g & $1380(620)$ & $1440(610)$ & 0.6 \\
\hline CRIB score & $7(4)$ & $6(4)$ & 0.17 \\
\hline Small for gestational age, $\mathrm{n}(\%)$ & $10(19)$ & $10(14)$ & 0.79 \\
\hline Surfactant use, $\mathrm{n}(\%)$ & $45(81)$ & $58(84)$ & 0.98 \\
\hline Pneumothorax, $\mathrm{n}(\%)$ & $4(7)$ & $1(1)$ & 0.13 \\
\hline Grade 3-4 IVH, $\mathrm{n}(\%)$ & $6(11)$ & $10(14)$ & 0.93 \\
\hline Periventricular leukomalacia, $\mathrm{n}(\%)$ & $2(4)$ & $6(9)$ & 0.28 \\
\hline Necrotizing enterocolitis, $\mathrm{n}(\%)$ & $2(4)$ & $6(9)$ & 0.68 \\
\hline Died during study period, $\mathrm{n}(\%)$ & $5(9)$ & $8(11)$ & 0.49 \\
\hline
\end{tabular}

Data are means (SD) or numbers (n) and percentages. IVH: Intraventricular hemorrhage.

Birth weight, gestational age and CRIB score were comparable between the two groups at the time of admission.

intervention (22\%) and 310 times of the 1,380 scores after intervention (also 22\%).

When the target range of 18 to 24 was not reached, the nursing staff more often adjusted the analgesic/ sedative dosages after intervention ( $53 \%$ vs. $34 \%, p<0.001)$.

The percentage of patients who received analgesia/ sedation during invasive ventilation was comparable in the two groups: $66 \%$ vs. $76 \%, p=0.23$. The mean hourly

Table 2 Changes in pain and discomfort (PAD) evaluation and management and other parameters of NICU hospitalization, before (Control) and after (Intervention) inclusion of the PAD module

\begin{tabular}{|c|c|c|c|}
\hline & Control & Intervention & \\
\hline & $n=53$ & $n=69$ & $p$ \\
\hline $\begin{array}{l}\text { Infants whose pain was assessed } \\
\text { daily, } \mathrm{n}(\%)\end{array}$ & $34(64)$ & $61(88)$ & 0.002 \\
\hline Number of assessments prescribed daily & $1(2)$ & $3(2)$ & $<0.01$ \\
\hline Number of assessments performed daily & $1(1)$ & $3(1)$ & $<0.01$ \\
\hline \multicolumn{4}{|l|}{ COMFORT score } \\
\hline -Number of scores & 813 & 1380 & \\
\hline -Mean value & $20(3)$ & $19(3)$ & 0.07 \\
\hline $\begin{array}{l}\text {-Scores outside the target range, } \\
\mathrm{n}(\%)\end{array}$ & $182(22)$ & $310(22)$ & 0.96 \\
\hline \multicolumn{4}{|l|}{ Absence of drug adaptation by the nurse } \\
\hline $\begin{array}{l}\text {-For scores outside the target } \\
\text { range, } \mathrm{n}(\%)\end{array}$ & $119(15)$ & $145(10)$ & $<0.01$ \\
\hline -For scores >24, n (\%) & $11(2)$ & $6(0.5)$ & 0.04 \\
\hline -For scores $<18, \mathrm{n}(\%)$ & $108(13)$ & $139(9.5)$ & 0.03 \\
\hline Nosocomial infection, n (\%) & $13(25)$ & $20(29)$ & 0.61 \\
\hline Length of invasive ventilation, days & $7(9)$ & $8(11)$ & 0.59 \\
\hline Length of stay in the NICU, days & $23(17)$ & $20(20)$ & 0.38 \\
\hline
\end{tabular}

Data are means (SD) or numbers ( $n$ ) and percentages.
Table 3 Analgesic and sedative drugs and their potential side effects, before (Control) and after (Intervention) inclusion of the pain and discomfort module

\begin{tabular}{|c|c|c|c|}
\hline & Control & Intervention & \\
\hline & $n=53$ & $n=69$ & $p$ \\
\hline $\begin{array}{l}\text { Patients with sedatives or analgesics, } \\
\mathrm{n}(\%)\end{array}$ & $35(66)$ & $52(76)$ & 0.23 \\
\hline Sufentanil, $\mu \mathrm{g} / \mathrm{kg} / \mathrm{hr}$ & $0.16(0.1)$ & $0.16(0.08)$ & 1 \\
\hline Midazolam, $\mu \mathrm{g} / \mathrm{kg} / \mathrm{hr}$ & $31(18)$ & $47(45)$ & 0.016 \\
\hline $\begin{array}{l}\text { Fluid bolus and/or vasoactive drugs, } \\
\mathrm{n}(\%)\end{array}$ & $10(19)$ & $13(19)$ & 1 \\
\hline Preventive treatment of WS, $\mathrm{n}(\%)$ & $20(38)$ & $20(29)$ & 0.46 \\
\hline
\end{tabular}

dose of sufentanil was not modified after the intervention, whereas midazolam was higher. This change was not associated with hypotension significant enough to require fluid boluses and/or vasoactive drugs. A strategy to prevent withdrawal syndrome was implemented for 20 infants in each study period, and no withdrawal syndrome was observed over the entire study (Table 3).

Pain module inclusion and changes in the parameters of NICU hospitalization

The duration of invasive mechanical ventilation, the duration of the NICU stay, and the rates of nosocomial infection and mortality remained comparable before and after intervention (Table 2).

\section{Discussion}

This study showed that adding a PAD module to a NICU CPOE system improved both PAD evaluation and analgesia/sedation dose adaptation on the basis of the scores. The midazolam dosage was increased after module inclusion, whereas the durations of ventilation and NICU stay were not affected.

The evaluation of PAD is mandatory in France and is part of the nursing role (Decree of competence No. 2004-802 of July 29, 2004, regarding professional nursing acts and the exercise of the nursing profession). Yet despite this, PAD evaluation remains inadequate on intensive care units-whether neonatal, pediatric, or adult-with the prevalence of patients evaluated ranging from $40 \%$ to $70 \%[7,19,20]$. Including a specific module in our CPOE system thus seemed to be a potential means to improve the evaluation rate for our nursing staff. For the physicians, a daily suggestion to validate an algorithm for adapting analgesia/sedation dosages on the basis of COMFORT scores was expected to lead to systematic discussions with the nurses on the PAD of the individual patient. For the nurses, the immediate association of a score with a therapeutic response was expected to reinforce the importance of regular PAD 


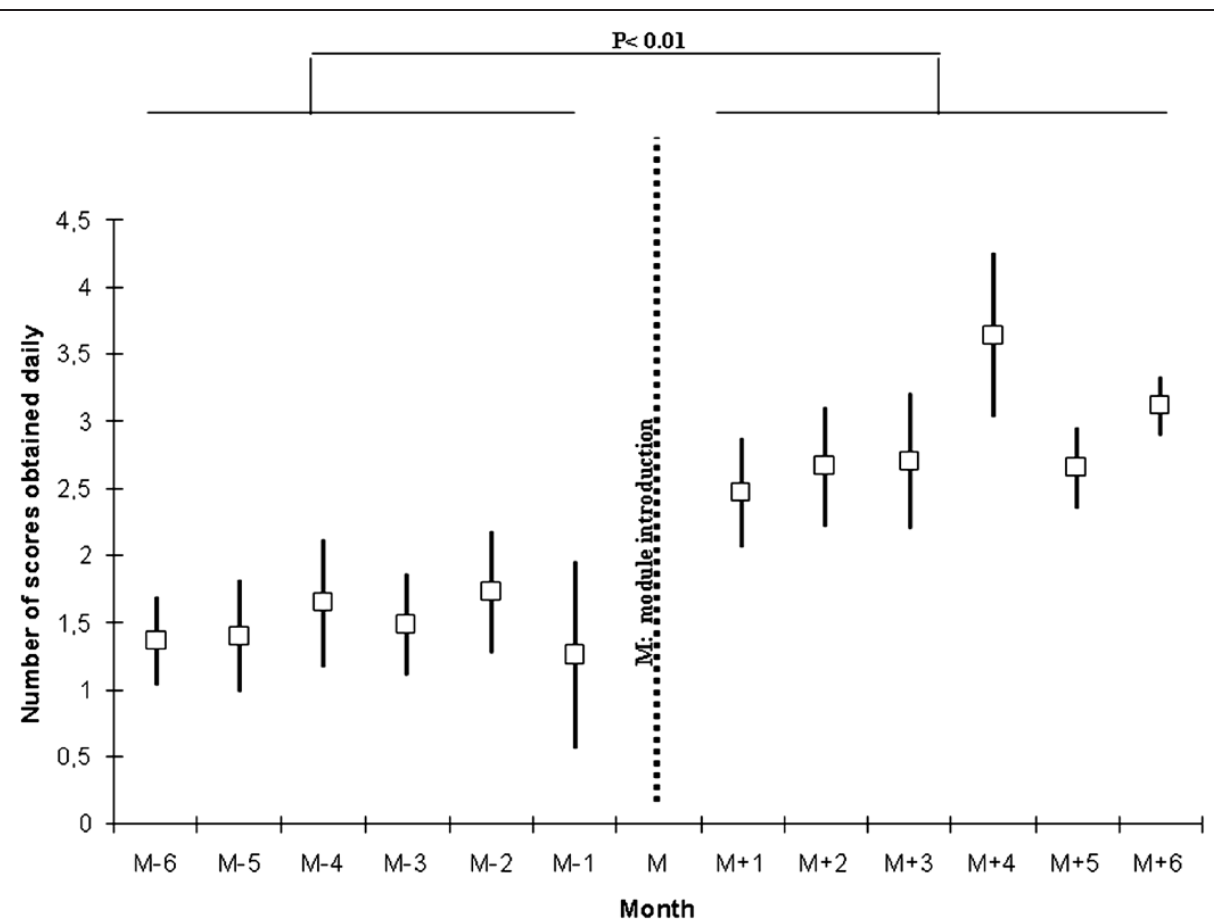

Figure 2 Number of individual daily COMFORT scores during mechanical invasive ventilation, before and after module inclusion. Data are means and SD.

evaluation [21,22]. Nurses feel supported in performing these evaluations when they know that the physicians are strongly involved [23]. The inclusion of the module improved drug adaptation by the nurses, whether the scores were high or low. However, in both periods, the nurses were less inclined to act when scores were low. This result points to the need to transmit a balanced message on PAD management by also emphasizing the risks associated with excessive sedation and analgesia.

The durations of invasive ventilation and NICU hospitalization and the rate of nosocomial infection remained comparable before and after the intervention. The literature has shown conflicting results regarding the impact of an analgesia/sedation algorithm on the duration of invasive ventilation [24,25]. Only certain studies on adult patients have demonstrated a shorter ventilation period [21,23,26-28] or a benefit in terms of the pneumonia associated with mechanical ventilation [26]. However, the protocols for adult ICUs include daily interruption of analgesia/sedation to determine whether the patient can be extubated $[28,29]$. This strategy, which may in itself reduce the time of ventilation, is rarely used in pediatrics [30] and was not employed on our unit. In any case, the small number of subjects in our study did not permit any conclusions to be drawn regarding this secondary outcome. Moreover, the algorithm for evaluation and management of PAD was the same during the two study periods. We studied only the systematization of this algorithm and not its implementation.

We chose the COMFORT scale over other multidimensional PAD scales, because it has been validated for use in newborns to assess objectively the adequacy of analgesia/sedation and it is widely used [13,19,31]. However, as opposed to the situation for adults, no scale is currently able to distinguish pain from discomfort in newborns [32,33]. This lack of specificity in the COMFORT scale implies a certain degree of subjectivity in the interpretation of scores, and frequent discussions between physicians and nurses are needed to determine the best therapeutic choice as pain and discomfort are managed differently. As noted in the Methods section, we optimized the environmental conditions before proceeding to a therapeutic adaptation of either midazolam if the infant was assessed as "uncomfortable or agitated" or sufentanil if assessed as "hyperalgesic." It was noteworthy that the more systematized evaluation of PAD, following the addition of the module, led to an increase in the prescription of sedatives and not analgesics. One explanation is that our NICU staff has become very sensitized to pain management since 2002. When a newborn is exposed to a situation likely to generate pain, evaluation and management of the pain is nearly systematic. In contrast, our results indicated that situations of discomfort were underestimated by the nursing staff and that multidimensional scales might help to better 
sensitize them to discomfort and its management, through medication or other means.

Whether the use of sedation and analgesia is recommended during invasive ventilation in newborns, the use of midazolam remains controversial [34]. Nevertheless, this drug is widely used on French NICUs, and no adverse effect was found at the age of 5 years in a large cohort of premature infants exposed to extended sedation/analgesia compared with an unexposed group [35].

The limitations of the study include the pre-post design. The CPOE system had been in use on our unit before the study began, and thus randomization with and without a computerized tool was not feasible. We therefore used a simple before-after design for the pain and discomfort module. However, this method is open to criticism and did not allow us to affirm that our results were the exclusive consequence of module introduction. Alternative explanations include unnoticed changes in the patient characteristics at inclusion or in the management protocols on the NICU. The nursing staff turnover was $6 \%$ during the study, and we also cannot exclude that some of the "new" nurses had undertaken personal training on pain. Indeed, these nurses were generally young and perhaps more sensitized to pain management. With a short period of observation, only 6 months after module introduction, we cannot assume that the observed changes were sustained over time. The limited number of infants, the monocentric nature of the study, and the specificities of our "homemade" module also are limitations for extrapolating our data to other NICUs. Nevertheless, we believe that our results will serve to encourage reflection on how to improve PAD evaluation in critically ill newborns.

\section{Conclusions}

The addition of a PAD module to an NICU CPOE system seems to have been an effective means to improve the systematic evaluation of PAD in premature newborns under invasive ventilation. Further studies at a bigger scale are now needed to validate this type of resource in the daily management of PAD in neonatology.

\section{Abbreviations \\ CPOE: computerized physician order entry; GA: gestational age;} NICU: neonatal intensive care unit; PAD: pain and discomfort.

\section{Competing interests}

The authors declare that they have no competing interests.

\section{Authors' contributions}

All authors are legitimate and have actively contributed to the work as follows: NM and CM: experts in our team for pain and discomfort management: study design, data collection, and manuscript preparation. RC: international expert in pain assessment and management: technical advice about the study design, manuscript preparation, review, and correction. RM: developed the computerized physician order entry system and the pain and discomfort module on our unit. CC and ARBN: contribution to the methodological part of the study design, monitoring, management of the data, statistical analysis of the data, and reviewing the manuscript. GC: study design and interpretation of the data, wrote the paper, and is the corresponding author. All authors read and approve the final manuscript.

\section{Authors' information}

This work was carried out in the Neonatal Intensive Care Unit (NICU) of Arnaud de Villeneuve Hospital, CHU Montpellier, F-34000 France.

\section{Author details}

${ }^{1}$ Neonatology Department, Arnaud de Villeneuve Hospital, CHU Montpellier, Montpellier F-34000, France. ${ }^{2}$ Pediatric Emergency Unit, Armand Trousseau Hospital, Assistance Publique-Hôpitaux de Paris, Paris F-75000, France. ${ }^{3}$ INSERM U953 Pierre and Marie Curie University, Paris, France. ${ }^{4}$ Neonatology and Intensive Care Unit, Montpellier University Hospital Centre, Arnaud de Villeneuve Hospital, 371 Avenue du Doyen G Giraud, 34295, Montpellier, Cedex 5, France.

Received: 2 March 2012 Accepted: 2 July 2012

Published: 22 August 2012

\section{References}

1. Carbajal R, Rousset A, Danan C, Coquery S, Nolent P, Ducroca S, Saizou C, Lapillonne A, Granier M, Durand P, Lenclen R, Coursol A, Hubert P, de Saint Blanquat L, Boëlle PY, Annequin D, Cimerman P, Anand KJ, Bréart G: Epidemiology and treatment of painful procedures in neonates in intensive care units. JAMA 2008, 300:60-70.

2. Simons $\mathrm{SH}$, van Dijk M, Anand KS, Roofthooft D, van Lingen RA, Tibboel D: Do we still hurt newborn babies? A prospective study of procedural pain and analgesia in neonates. Arch Pediatr Adolesc Med 2003, 157:1058-1064.

3. Guinsburg R, Kopelman BI, Anand KJ, de Almeida MF, Peres Cde A, Miyoshi $\mathrm{MH}$ : Physiological, hormonal, and behavioral responses to a single fentanyl dose in intubated and ventilated preterm neonates. J Pediatr 1998, 132:954-959.

4. Grunau RE, Tu MT, Whitfield MF, Oberlander TF, Weinberg J, Yu W, Thiessen P, Gosse G, Scheifele D: Cortisol, behavior, and heart rate reactivity to immunization pain at 4 months corrected age in infants born very preterm. Clin J Pain 2010, 26:698-704

5. Johnston CC, Stevens BJ: Experience in a neonatal intensive care unit affects pain response. Pediatrics 1996, 98:925-930.

6. Grunau RE, Holsti L, Haley DW, Oberlander T, Weinberg J, Solimano A, Whitfield MF, Fitzgerald C, Yu W: Neonatal procedural pain exposure predicts lower cortisol and behavioral reactivity in preterm infants in the NICU. Pain 2005, 113:293-300.

7. Debillon T, Bureau V, Savagner C, Zupan-Simunek V, Carbajal R, French National Federation of Neonatologists: Pain management in French neonatal intensive care units. Acta Paediatr 2002, 91:822-826.

8. Taddio A, Pulleyblank R, Stephens D, McNair C, Shah V: Canadian neonatologist practices regarding opioid use in ventilated and spontaneously breathing infants undergoing medical procedures. Clin J Pain 2010, 26:422-428.

9. Sharek PJ, Powers R, Koehn A, Anand KJ: Evaluation and development of potentially better practices to improve pain management of neonates. Pediatrics 2006, 118(Suppl 2):78-86.

10. 3rd Dunbar AE, Sharek PJ, Mickas NA, Coker KL, Duncan J, McLendon D, Pagano C, Puthoff TD, Reynolds NL, Powers RJ, Johnston CC: Implementation and case-study results of potentially better practices to improve pain management of neonates. Pediatrics 2006, 118(Suppl 2):87-94.

11. Ambuel B, Hamlett KW, Marx CM, Blumer JL: Assessing distress in pediatric intensive care environments: the COMFORT scale. J Pediatr Psychol 1992, 17:95-109.

12. van Dijk M, de Boer JB, Koot HM, Tibboel D, Passchier J, Duivenvoorden HJ: The reliability and validity of the COMFORT scale as a postoperative pain instrument in 0 to 3-year-old infants. Pain 2000, 84:367-377.

13. Wielenga JM, De Vos R, de Leeuw R, De Haan RJ: COMFORT scale: a reliable and valid method to measure the amount of stress of ventilated preterm infants. Neonatal Netw 2004, 23:39-44. 
14. Marx CM, Smith PG, Lowrie LH, Hamlett KW, Ambuel B, Yamashita TS, Blumer JL: Optimal sedation of mechanically ventilated pediatric critical care patients. Crit Care Med 1994, 22:163-170.

15. Holsti L, Grunau RE, Oberlander TF, Whitfield MF: Specific Newborn Individualized Developmental Care and Assessment Program movements are associated with acute pain in preterm infants in the neonatal intensive care unit. Pediatrics 2004, 114:65-72.

16. Kaplan JM, Ancheta R, Jacobs BR: Clinical Informatics Outcomes Research Group. Inpatient verbal orders and the impact of computerized provider order entry. J Pediatr 2006, 149:461-467.

17. Szymankiewicz M, Vidyasagar D, Gadzinowski J: Predictors of successful extubation of preterm low-birth-weight infants with respiratory distress syndrome. Pediatr Crit Care Med 2005, 6:44-49.

18. Finnegan LP, Connaughton JF Jr, Kron RE, Emich JP: Neonatal abstinence syndrome assessment and management. Addict Dis 1975, 2:141-158.

19. Jin HS, Yum MS, Kim SL, Shin HY, Lee EH, Ha EJ, Hong SJ, Park SJ: The efficacy of the COMFORT scale in assessing optimal sedation in critically ill children requiring mechanical ventilation. J Korean Med Sci 2007, 22:693-697.

20. Payen JF, Chanques G, Mantz J, Hercule C, Auriant I, Lequillou JL, Binhas M, Genty C, Rolland C, Bosson JL: Current practices in sedation and analgesia for mechanically ventilated critically ill patients: a prospective multicenter patient-based study. Anesthesiology 2007, 106:687-695.

21. Chanques G, Jaber S, Barbotte E, Violet S, Sebbane M, Perrigault PF, Mann C, Lefrant JY, Eledjam JJ: Impact of systematic evaluation of pain and agitation in an intensive care unit. Crit Care Med 2006, 34:1691-1699.

22. Allegaert K, Veyckemans F, Tibboel D: Clinical practice: analgesia in neonates. Eur J Pediatr 2009, 168:765-770.

23. De Jonghe B, Bastuji-Garin S, Fangio P, Lacherade JC, Jabot J, Appéré-De-Vecchi C, Rocha N, Outin H: Sedation algorithm in critically ill patients without acute brain injury. Crit Care Med 2005, 33:120-127.

24. Elliott R, McKinley S, Aitken LM, Hendrikz J: The effect of an algorithm-based sedation guideline on the duration of mechanical ventilation in an Australian intensive care unit. Intensive Care Med 2006, 32:1506-1514

25. Bucknall TK, Manias E, Presneill JJ: A randomized trial of protocol-directed sedation management for mechanical ventilation in an Australian intensive care unit. Crit Care Med 2008, 36:1444-1450.

26. Quenot JP, Ladoire S, Devoucoux F, Doise JM, Cailliod R, Cunin N, Aubé H, Blettery B, Charles PE: Effect of a nurse-implemented sedation protocol on the incidence of ventilator-associated pneumonia. Crit Care Med 2007, 35:2031-2036.

27. Brook AD, Ahrens TS, Schaiff R, Prentice D, Sherman G, Shannon W, Kollef $\mathrm{MH}$ : Effect of a nursing-implemented sedation protocol on the duration of mechanical ventilation. Crit Care Med 1999, 27:2609-2615

28. Kress JP, Pohlman AS, O'Connor MF, Hall JB: Daily interruption of sedative infusions in critically ill patients undergoing mechanical ventilation. N Engl J Med 2000, 342:1471-1477.

29. Girard TD, Kress JP, Fuchs BD, Thomason JW, Schweickert WD, Pun BT, Taichman DB, Dunn JG, Pohlman AS, Kinniry PA, Jackson JC, Canonico AE, Light RW, Shintani AK, Thompson JL, Gordon SM, Hall JB, Dittus RS, Bernard GR, Ely EW: Efficacy and safety of a paired sedation and ventilator weaning protocol for mechanically ventilated patients in intensive care (Awakening and Breathing Controlled trial): a randomised controlled trial. Lancet 2008, 371:126-134.

30. Leclerc F, Noizet O, Botte A, Binoche A, Chaari W, Sadik A, Riou Y: Weaning from invasive mechanical ventilation in pediatric patients (excluding premature neonates). Arch Pediatr 2010, 17:399-406.

31. Hummel $P$, van Dijk M: Pain assessment: current status and challenges. Semin Fetal Neonatal Med 2006, 11:237-245.

32. Anand KJ, Aranda JV, Berde CB, Buckman S, Capparelli EV, Carlo W, Hummel P, Johnston CC, Lantos J, Tutag-Lehr V, Lynn AM, Maxwell LG, Oberlander TF, Raju TN, Soriano SG, Taddio A, Walco GA: Summary proceedings from the neonatal pain-control group. Pediatrics 2006, 117:S9-S22.

33. Ely EW, Truman B, Shintani A, Thomason JW, Wheeler AP, Gordon S, Francis J, Speroff T, Gautam S, Margolin R, Sessler CN, Dittus RS, Bernard GR: Monitoring sedation status over time in ICU patients: reliability and validity of the Richmond Agitation-Sedation Scale (RASS). JAMA 2003, 289:2983-2991.
34. Bellù $R$, de Waal KA, Zanini R: Opioids for neonates receiving mechanical ventilation. Cochrane Database Syst Rev 2008, 1:CD004212.

35. Rozé JC, Denizot S, Carbajal R, Ancel PY, Kaminski M, Arnaud C, Truffert P, Marret S, Matis J, Thiriez G, Cambonie G, André M, Larroque B, Bréart G: Prolonged sedation and/or analgesia and 5-year neurodevelopment outcome in very preterm infants. Arch Pediatr Adolesc Med 2008, 162:728-733.

doi:10.1186/2110-5820-2-38

Cite this article as: Mazars et al.: Implementation of a neonatal pain management module in the computerized physician order entry system. Annals of Intensive Care 2012 2:38.

\section{Submit your manuscript to a SpringerOpen ${ }^{\odot}$ journal and benefit from:}

- Convenient online submission

- Rigorous peer review

- Immediate publication on acceptance

- Open access: articles freely available online

- High visibility within the field

- Retaining the copyright to your article

Submit your next manuscript at $>$ springeropen.com 